\title{
Optical properties of a light-emitting polymer directly patterned by soft lithography
}

\author{
J. R. Lawrence \\ Ultrafast Photonics Collaboration \& Organic Semiconductor Centre, School of Physics \& Astronomy, \\ University of St. Andrews, North Haugh, St. Andrews, Fife KY16 9SS, United Kingdom \\ P. Andrew and W. L. Barnes \\ Thin Film Photonics Group, School of Physics, University of Exeter, Stocker Road, Exeter, Devon, \\ EX4 4QL, United Kingdom \\ M. Buck \\ Organic Semiconductor Centre \& School of Chemistry, University of St. Andrews, North Haugh, \\ St. Andrews, Fife KY16 9ST, United Kingdom \\ G. A. Turnbull and I. D. W. Samuel ${ }^{\text {a) }}$ \\ Ultrafast Photonics Collaboration \& Organic Semiconductor Centre, School of Physics \& Astronomy, \\ University of St. Andrews, North Haugh, St. Andrews, Fife KY16 9SS, United Kingdom
}

(Received 19 February 2002; accepted for publication 29 June 2002)

\begin{abstract}
We present the optical properties of a directly patterned light-emitting polymer. The patterned poly(2-methoxy-5-(3',7'-dimethyloctyloxy)-paraphenylenevinylene film is fabricated using hot embossing lithography. The effect of the embossed microstructure on the light emitted from the polymer is examined by measuring the angle-dependent photoluminescence and its photonic band structure. The imposed grating modifies the emitted light by Bragg scattering into free space light that would otherwise be trapped as waveguide modes. This simple patterning technique may find application in improving the performance of light-emitting polymer devices. (C) 2002 American Institute of Physics. [DOI: 10.1063/1.1502187]
\end{abstract}

Semiconducting conjugated polymers are a very promising class of materials for use in optoelectronics. They are easily-processible and are suitable for a range of applications due to their high photoluminescence (PL) quantum yield, large gain cross section, broad emission spectrum and their ability to be electrically pumped. ${ }^{1-3}$ The performance of lasers, light emitting diodes, and displays made from these materials can be further improved by incorporating wavelength scale microstructures. ${ }^{4}$ At present such microstructured devices are made using one or a combination of complex techniques such as photolithography, electron beam lithography, and chemical etching. New fabrication methods that retain the ease of processing that is a key advantage of polymeric materials would therefore be very attractive.

In this letter we use a simple technique known as hot embossing lithography ${ }^{5,6}$ to fabricate sub-wavelength scale microstructures in a light-emitting polymer film. We measure angle-dependent PL to show how the microstructure affects the light emission from the film and explain the results by measuring the photonic band structure and by theoretical modeling.

The conjugated polymer used in this study is poly[ $(2-$ methoxy-5-(3',7'-dimethyloctyloxy)-1,4 paraphenylenevinylene] $\left(\mathrm{OC}_{1} \mathrm{C}_{10}-\mathrm{PPV}\right)$ and its structure is shown in Fig. 1. Thin films of $\mathrm{OC}_{1} \mathrm{C}_{10}$-PPV were formed by spin coating from chlorobenzene onto flexible plastic substrates (overhead transparencies). This results in films of about $100 \mathrm{~nm}$ in thickness. These films are then patterned using a soft litho-

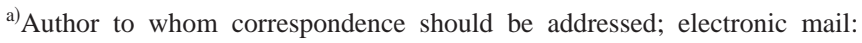
idws@st-andrews.ac.uk
}

graphic technique known as hot embossing similar to the method used to microstructure a photodiode. ${ }^{6}$ The polymer was heated to $20^{\circ} \mathrm{C}$ above its glass transition temperature $\left(T_{g} \sim 200^{\circ} \mathrm{C}^{7}\right)$, that is, the temperature at which it begins to soften and flow. A master copy of the desired structure was pressed into the polymer with a pressure of $48 \mathrm{~N} / \mathrm{cm}^{2}$. The pressure needed for embossing was relatively low compared to other embossing methods. In order to prevent the $\mathrm{OC}_{1} \mathrm{C}_{10}$ - PPV from sticking, the silica master was first coated with a monolayer of octadecyltrichlorosilane. Under pressure from the master, the polymer indents and flows around the master conforming to its shape. After $20 \mathrm{~min}$ the polymer was allowed to cool with the master still in place. This cooling allows the polymer to drop below its glass transition temperature effectively "freezing in" the imprinted structure. The master was then removed from the polymer leaving the replicated structure embossed into the polymer.

In the present work the master structures were crossed gratings formed in photoresist by multiple exposure to interfering laser beams and etched into silica as shown in Fig. 2(a). The angle $\phi$ between the two gratings was $90^{\circ}$, and each grating had a period of $400 \mathrm{~nm}$ and a peak-to-trough depth of $30 \mathrm{~nm}$. The entire procedure was carried out in air. In order to characterize the embossed structure, the patterned polymer films were examined using an atomic force microscope. An image of the embossed $\mathrm{OC}_{1} \mathrm{C}_{10}$-PPV film obtained using atomic force microscopy is shown in Fig. 1. The imprinted crossed grating structure can be clearly seen in the film. The embossed structure has a period of $400 \mathrm{~nm}$ and a depth of $20 \mathrm{~nm}$. The period matches exactly that of the etched silica master but the embossed grating is shallower. 

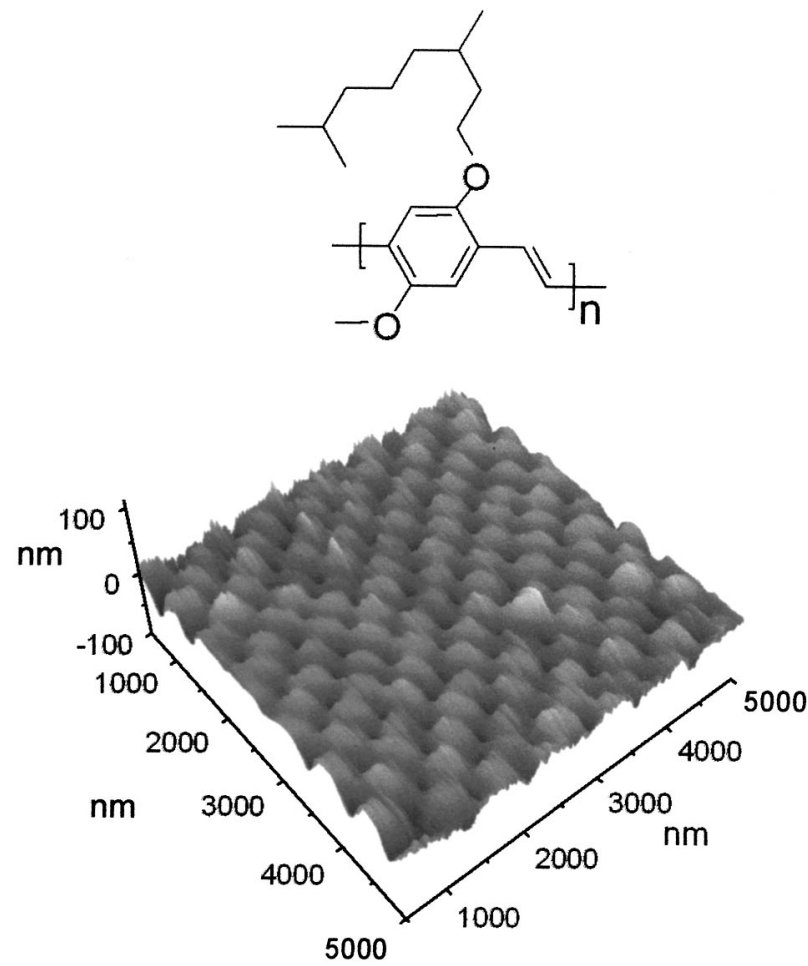

FIG. 1. An atomic force microscope image and chemical structure of the $\mathrm{OC}_{1} \mathrm{C}_{10}$-PPV film patterned by hot embossing.

In order to understand how the microstructure affects the light emission, the photonic mode structure of the film was measured by angle-dependent transmission as shown in Fig. 2(b). ${ }^{8,9}$ The impact of the embossed microstructure on the photonic band structure is shown in Fig. 3. Figure 3(a) shows the modes observed when the incident beam is $s$-polarized and Fig. 3(b) shows p-polarization. When the incident light is $s$-polarized, two dark bands are observed, both starting at $\omega=1.63 \mu \mathrm{m}^{-1}$ and ending at 1.55 and $1.7 \mu \mathrm{m}^{-1}$, respectively. For $p$-polarized incident light there is a single horizontal dark band showing little dispersion and centered at $\omega=1.63 \mu \mathrm{m}^{-1}$. These dark bands correspond to a drop in transmission, which indicates that the incident light has been coupled into a waveguide mode. The incident light is diffracted by the periodic microstructure at an angle greater

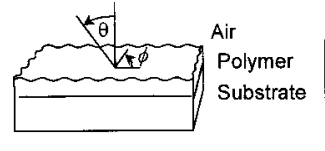

(a)

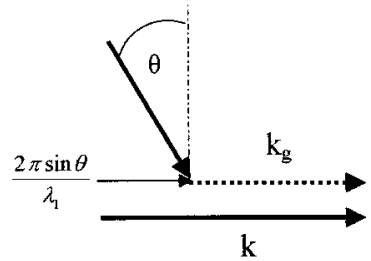

(c)

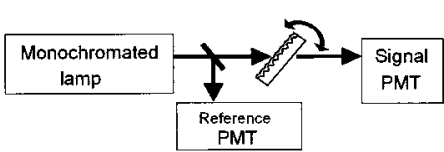

(b)

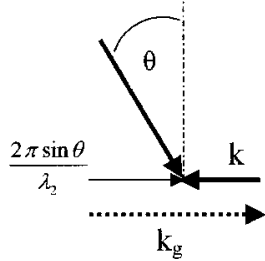

(d)
FIG. 2. (a) Polymer film with imprinted crossed grating. The plane of incidence was aligned parallel to one of the sets of gratings. (b) Experimental setup for measurement of photonic mode structure. Bragg scattering when the magnitude of the incident wave vector $k$ is (c) greater than and (d) less

than that of the grating vector $k_{g}$.
Downloaded 18 Mar 2005 to 144.173.6.75. Redistribution subject to AIP license or copyright, see http://apl.aip.org/apl/copyright.jsp

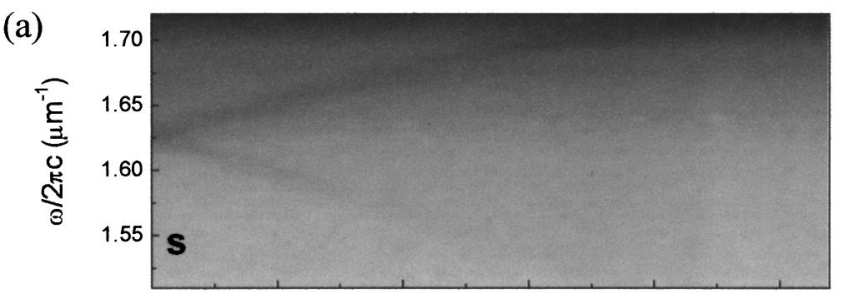

(b)

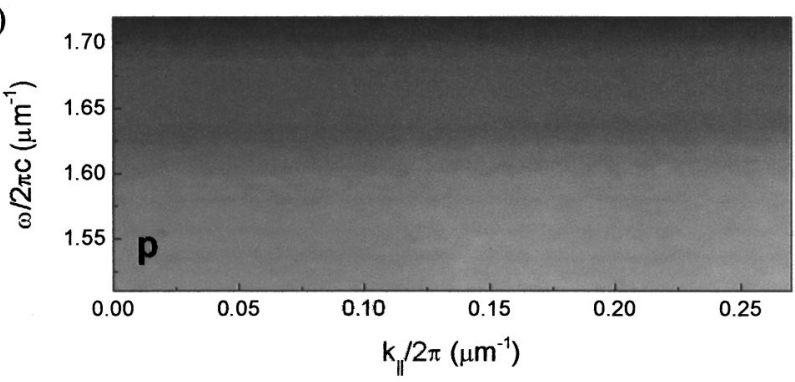

FIG. 3. Measured photonic mode structure of directly patterned $\mathrm{OC}_{1} \mathrm{C}_{10}$-PPV film when probed with (a) $s$ and (b) $p$-polarized light.

than the critical angle, thereby causing the light to waveguide inside the film. In Fig. 3(a) it can be seen that for each incident polar angle there are two wavelengths that are strongly scattered by the microstructure. This behavior is explained by Fig. 2. The scattering process of the grating can be thought of as adding a wave vector $\left(k_{g}= \pm 2 \pi / \Lambda\right)$ to the optical modes of the waveguide $\left(k=2 \pi n_{\text {eff }} / \lambda\right)$, where $\Lambda$ is the period of the corrugation, $n_{\text {eff }}$ is the effective refractive index of the waveguide, and $\lambda$ is the wavelength of the incident light. When a light beam is directed onto the guide from free space at a polar angle of $\theta$ to the substrate normal, the field has an in-plane wave vector component of $(2 \pi \sin \theta) / \lambda$. If this component matches the difference $\left|k-k_{g}\right|$, light will be strongly coupled into the waveguide mode. For each angle $\theta$ there are two wavelengths for which this condition is satisfied: $|k|>\left|k_{g}\right|$ and $|k|<\left|k_{g}\right|$, as illustrated in Figs. 2(c) and $2(\mathrm{~d})$.

Having measured the photonic band structure of the film, we now examine the effects of the microstructure on the light emission. To investigate this we made measurements of the angle-dependent PL. The patterned polymer film was housed in a circular vacuum chamber and excited using the second harmonic output of a diode pumped continuous wave $\mathrm{Nd}$ :YAG laser $(532 \mathrm{~nm})$. Light emitted from the film was collected by an optical fiber and detected using a CCD spectrometer. The end of the optical fiber was attached to a freerotating moveable arm mounted on the vacuum chamber to enable light emitted over a wide range of angles to be detected.

Figure 4(a) shows the PL spectrum of the directly patterned $\mathrm{OC}_{1} \mathrm{C}_{10}$-PPV film measured at various angles. As the angle of observation ( $\theta$ in Fig. 2(a)) changes, the central peak splits into two peaks which move to longer and shorter wavelengths due to Bragg scattering. ${ }^{10,11}$ This can be seen more clearly in Fig. 4(b) where each PL peak is plotted as a function of its wavelength and angle of observation. All scattered features shown in Fig. 4(b) originate from emission into the $\mathrm{TE}_{0}$ mode of the waveguide (shown in Fig. 3), which is then scattered by one of the crossed gratings. The feature indicated by the squares is due to light coupling to the waveAIP license or copyright, see http://apl.aip.org/apl/copyright.jsp 


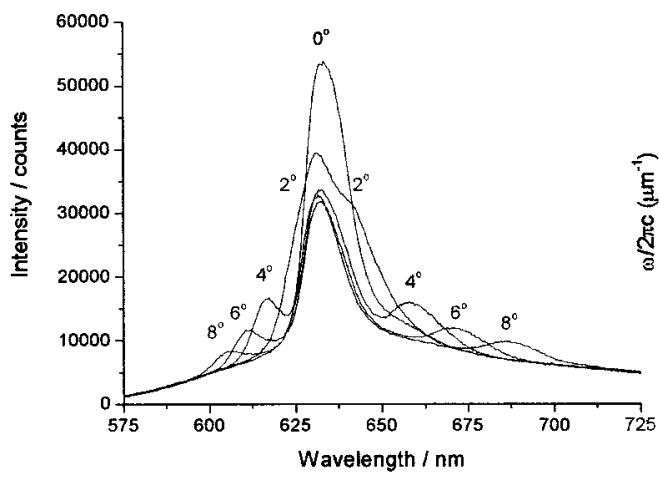

(a)

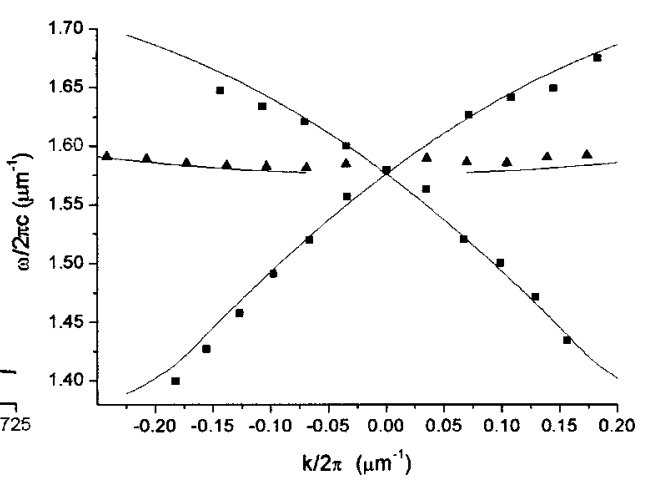

(b)
FIG. 4. (a) Angle dependent PL spectrum of a directly patterned $\mathrm{OC}_{1} \mathrm{C}_{10}$-PPV film and (b) PL peak positions as a function of in-plane wavevector and frequency (points) and theoretical fit (lines). guide mode via Bragg scattering arising from the grating that has grooves running perpendicular to the plane of observation, that is, Bragg vector in plane of incidence. The feature indicated by the triangles is due to light coupling to the waveguide mode via Bragg scattering arising from the grating that has grooves parallel to the observation plane. The frequencies and in-plane wavevectors for optimum coupling in these two cases can be given by Eqs. (1) and (2), respectively

$$
\begin{aligned}
& \omega= \pm k \pm m k_{g}= \pm \frac{2 \pi n_{\mathrm{eff}}}{\lambda} \pm m \frac{2 \pi}{\Lambda}, \\
& \omega= \pm \sqrt{k^{2}-\left(m k_{g}\right)^{2}}= \pm \sqrt{\left(\frac{2 \pi n_{\mathrm{eff}}}{\lambda}\right)^{2}-\left(m \frac{2 \pi}{\Lambda}\right)^{2}},
\end{aligned}
$$

where $\Lambda$ is the fundamental grating period and $m$ is an integer. These equations are used to fit the experimental data. The fit is shown as a solid line in Fig. 4(b). The refractive index of $\mathrm{OC}_{1} \mathrm{C}_{10}-\mathrm{PPV}$ as a function of wavelength was measured by spectroscopic ellipsometery. Using this information, $n_{\text {eff }}$ the effective index of the waveguide could be calculated. ${ }^{12}$

Despite the relatively weak corrugation of the embossed structures when compared to that used when polymers are deposited onto microstructured substrates, ${ }^{4,13}$ significant modification of the PL spectra is still seen. This arises because the periodic modulation occurs at the polymer air interface, which has stronger index contrast than the polymer glass interface. We note a small discrepancy of $0.06 \mu \mathrm{m}^{-1}$ in the frequency of the modes observed in PL $\left(1.57 \mu \mathrm{m}^{-1}\right)$ and the photonic mode structure $\left(1.63 \mu \mathrm{m}^{-1}\right)$ in Fig. 3. It is likely that this arises from slightly different regions of the sample being measured, with the regions being of different thickness. A difference in thickness of less than $10 \%$ between the areas probed for each experiment would account for this discrepancy.

In summary, we have shown that directly patterning a conjugated polymer film provides a simple way of control- ling the emission of light from within it. The patterned film was examined by atomic force microscopy and shown to closely follow the shape of the master structure. The effect of the embossed microstructure on the emitted light was examined by comparing angle dependent PL with the photonic mode structure. It was found that the imposed grating enables light trapped in waveguide modes to be Bragg scattered out into free space. The observations also agree with waveguide mode structure deduced from measured refractive index data. Hot embossing processes such as this should lead to simpler device fabrication and provide flexibility in new device design.

We are grateful to EPSRC and SHEFC for financial support, and Covion for the supply of $\mathrm{OC}_{1} \mathrm{C}_{10}$-PPV. One of the authors (I. D. W. S.) is a Royal Society University Research Fellow.

${ }^{1}$ M. D. McGehee and A. J. Heeger, Adv. Mater. 12, 1655 (2000).

${ }^{2}$ R. H. Friend, R. W. Gymer, A. B. Holmes, J. H. Burroughes, R. N. Marks, C. Taliani, D. D. C. Bradley, D. A. Dos Santos, J. L. Bredas, M. Logdlund, and W. R. Salaneck, Nature (London) 397, 121 (1999).

${ }^{3}$ U. Scherf, S. Riechel, U. Lemmer, and R. F. Mahrt, Curr. Opin. Solid State Mater. Sci. 5, 143 (2001).

${ }^{4}$ J. M. Lupton, B. J. Matterson, M. J. Jory, W. L. Barnes, and I. D. W. Samuel, Appl. Phys. Lett. 77, 1 (2000).

${ }^{5}$ S. Y. Chou, P. R. Krauss, and P. J. Renstrom, Appl. Phys. Lett. 67, 3114 (1995).

${ }^{6}$ L. S. Roman, O. Inganas, T. Granlund, T. Nyberg, M. Svensson, M. R. Andersson, and J. C. Hummelen, Adv. Mater. 12, 189 (2000).

${ }^{7}$ T. Q. Nguyen, B. J. Scwartz, R. D. Schaller, J. C. Johnson, L. F. Lee, L. H. Haber, and R. J. Saykally, J. Phys. Chem. B 105, 5153 (2001).

${ }^{8}$ M. G. Salt and W. L. Barnes, Opt. Commun. 166, 151 (1999).

${ }^{9}$ M. G. Salt, W. L. Barnes, and I. D. W. Samuel, J. Mod. Opt. 48, 1085 (2001).

${ }^{10}$ B. J. Matterson, M. G. Salt, W. L. Barnes, and I. D. W. Samuel, Synth. Met. 101, 250 (1999)

${ }^{11}$ A. N. Safonov, M. Jory, J. M. Lupton, M. G. Salt, J. A. E. Wasey, W. L. Barnes, and I. D. W. Samuel, Synth. Met. 116, 145 (2001).

${ }^{12}$ A. Yariv, Quantum Electronics (John Wiley, New York, 1989).

${ }^{13}$ G. A. Turnbull, P. Andrew, M. J. Jory, W. L. Barnes, and I. D. W. Samuel, Phys. Rev. B 6412, 5122 (2001). 